\title{
Effet des conditions d'entreposage sur la dégradation de la couleur des dattes tunisiennes de type Deglet Nour
}

\author{
Mohsen ACHOUR*, Naoufel BAGGA
}

École supérieure des industries alimentaires de Tunis, 58 avenue Alain Savary, Tunis 1003, Tunisie achour_mohsen@yahoo.fr

${ }^{*}$ Correspondance et tirés à part

Fruits, 2005 , vol. 60 , p. $41-46$ (C) 2005 Cirad/EDP Sciences All rights reserved

DOI: $10.1051 /$ fruits:2005011

RESUMEN EsPAÑoL, p. 46

\section{Effect of storage conditions on the color degradation of Tunisian Deglet Nour dates.}

\begin{abstract}
Introduction. In Tunisia, Deglet Nour dates are famous for their gilded yellow color and they present a high commercial value; they constitute the majority of dates exported towards Europe. The aim of our work was to study effects of oxygen and temperature on the color degradation of this date type during the storage period. Materials and methods. The color evolution of Deglet Nour dates stored under a constant relative humidity (75\%), at three different temperatures $\left[(10,25 \text { and } 37)^{\circ} \mathrm{C}\right]$ and in the presence of three oxygen concentrations $[(0,20$ and 40$) \%]$ was followed each week for 35 days using the measurements of $L^{*}$ and $b^{*}$ parameters of the Hunter Lab scale. Results. The temperature played a determining part in the date color degradation during storage and the effect of oxygen was important, though secondary. Conclusion. Date color during storage proves to be able to be predicted starting from the only parameter $b^{*}$ of the Hunter Lab scale. In the case of dates conditioned under various oxygen concentrations and at various temperatures, the loss of the fruit's initial color was explained more by an ambient temperature increase than by the variation of the oxygen concentration of the storage environment. The degradation of the color would be caused by nonenzymatic and oxidative enzymatic tannings.
\end{abstract}

Tunisia / Phoenix dactylifera / dates / color / storage / biochemical reactions / biodegradation

Effet des conditions d'entreposage sur la dégradation de la couleur des dattes tunisiennes de type Deglet Nour.

Résumé - Introduction. En Tunisie, les dattes de type Deglet Nour sont réputées pour leur couleur jaune doré et elles présentent une valeur marchande élevée; elles constituent la majorité des dattes exportées vers l'Europe. L'objectif de nos travaux a été d'étudier l'effet de l'oxygène et de la température sur la dégradation de la couleur de ce type de dattes durant la période d'entreposage. Matériel et méthodes. L'évolution de la couleur de dattes du type Deglet Nour stockées sous humidité relative constante (75\%), à trois températures différentes $\left[(10,25 \text { et } 37)^{\circ} \mathrm{C}\right]$ et en présence de trois concentrations d'oxygène $[(0,20$ et 40$) \%]$ a été suivie chaque semaine durant 35 jours à l'aide de la mesure des paramètres $L^{*}$ et $b^{*}$ de l'échelle Hunter Lab. Résultats. La température a joué un rôle déterminant dans la dégradation de la couleur des dattes durant l'entreposage et l'effet de l'oxygène a été important quoique secondaire. Conclusion. La couleur des dattes au cours de leur stockage se révèle pouvoir être prédite à partir du seul paramètre $b^{*}$ de l'échelle Hunter Lab. Dans le cas des dattes conditionnées sous différentes concentrations en oxygène et à différentes températures, la perte de la couleur initiale du fruit a été davantage expliquée par une augmentation de la température ambiante que par la variation de la concentration en oxygène du milieu de conservation. La dégradation de la couleur serait causée par des brunissements non enzymatiques et enzymatiques oxydatifs.

Tunisie / Pboenix dactylifera / datte / couleur / stockage / réaction biochimique / biodégradation 


\section{Introduction}

La Tunisie est le premier pays exportateur de dattes dans le monde, offrant une moyenne annuelle de 35000 t pour une valeur dépassant $50 \mathrm{M} €$ [1]. Cette performance est en grande partie due à la haute valeur marchande de la variété Deglet Nour réputée pour sa couleur vive dorée, qui représente plus que $80 \%$ des exportations de dattes du pays. Cependant, lorsque la période d'entreposage est longue, la couleur spécifique de la datte Deglet Nour subit un brunissement qui causerait une dépréciation significative de sa qualité au moment de la commercialisation. Ce brunissement pourrait résulter de trois types de réactions [2] :

- une réaction faisant intervenir un composé à fonction carbonylée et un composé à groupement aminé (réaction de Maillard) : c'est un brunissement non enzymatique,

- une réaction aboutissant à un brunissement enzymatique,

- une réaction conduisant à un brunissement non enzymatique oxydatif mettant en jeu des tannins complexes.

La première réaction est particulièrement significative en présence de températures élevées. Les deux dernières réactions sont d'autant plus importantes que la concentration en oxygène du milieu est élevée. Une corrélation positive entre, d'une part, la teneur en eau des dattes et la température d'entreposage, d'autre part, le degré de brunissement a été expérimentalement démontrée [3]. Afin de diminuer la vitesse de brunissement, diverses techniques ont pu être utilisées : l'inactivation des enzymes (dénaturation par la température), le conditionnement à basse température ou le conditionnement sous vide ou sous atmosphère modifiée [4-6]. En particulier, et d'après les travaux récents de Calero [7] et Hong et Gross [8], l'utilisation du conditionnement sous atmosphère modifiée pourrait améliorer la conservation des fruits et légumes.

Afin d'aboutir à de meilleures conditions de conservation des dattes Deglet Nour aptes à leur maintenir une couleur attractive jusqu'à la commercialisation, leur couleur, exprimée à l'aide des paramètres de l'échelle Hunter Lab, a été suivie sur des échantillons conditionnés à une humidité relative constante et sous différentes combinaisons de températures et de concentrations en oxygène.

\section{Matériel et méthodes}

\subsection{Préparation des échantillons de dattes}

Des dattes de type Deglet Nour branchées ont été triées pour ne retenir que les fruits présentant une couleur vive dorée. Afin de contourner le problème de l'hétérogénéité de couleur pouvant être observée à la surface d'une même datte, nous avons étudié des zones de surface du fruit où la couleur était presque uniforme. Les échantillons ainsi sélectionnés ont été conditionnés dans des sachets en polyéthylène téréphtalate (PET) à l'aide d'une conditionneuse-soudeuse de type Minipack MVS 45.

Trois types de sachets ont été utilisés différant par la concentration en oxygène de leur contenu : $40 \%, 20 \%$ ou $0 \%$ (vide) de leur volume, puis les échantillons conditionnés ont été placés dans des dessiccateurs contenant une solution saline saturée de $\mathrm{NaCl}$ apte à assurer une humidité relative constante égale à $75 \%$. Trois dessiccateurs ont été utilisés pour tester trois températures de stockage différentes : $10{ }^{\circ} \mathrm{C}$ pour simuler une conservation des dattes à froid positif, $25^{\circ} \mathrm{C}$ pour simuler des conditions ambiantes standard de stockage et $37{ }^{\circ} \mathrm{C}$ pour simuler des conditions extrêmes d'entreposage.

\subsection{Mesure de la couleur}

La couleur des échantillons a été mesurée à l'aide d'un chromamètre Minolta CR-300 et exprimée selon l'échelle Hunter Lab qui permet de faire une bonne distinction parmi les couleurs saturées comme le sont celles des dattes. L'échelle présente trois paramètres :

- Le paramètre $L^{*}$ représente une approximation mathématique non linéaire de la réponse de l'oeil aux couleurs noires et blanches ; il a une valeur égale à 100 pour la couleur blanche et à 0 pour la couleur noir 
pur. Il donne une indication de la luminosité de la surface mesurée.

- Le paramètre $a^{*}$ prend des valeurs comprises entre -60 et +60 , les valeurs négatives indiquent les couleurs verdâtres et les valeurs positives les couleurs rougeâtres.

- Le paramètre $b^{*}$ prend également des valeurs entre -60 pour les couleurs jaunâtres à +60 pour les couleurs bleuâtres.

Sur cette base et du fait de la couleur des dattes qui varie du jaune doré au brun foncé, l'analyse des données dans le cadre de notre travail a été limitée aux paramètres $L^{*}$ et $b^{*}$ jugés significatifs pour l'évaluation de la dégradation de la couleur naturelle des dattes du type Deglet Nour tunisienne. Une mesure de la couleur initiale a été effectuée puis l'évolution de cette coloration au cours du stockage a été un suivie pendant 35 jours, à raison d'une mesure par semaine. L'évaluation de la couleur d'un échantillon a été réalisée hors sachet en plaçant les dattes sur un fond foncé et en effectuant trois répétitions par mesure.

\subsection{Compilation des résultats}

Afin de mieux apprécier l'évolution de la couleur des dattes au cours du temps et éviter la variabilité de la couleur initiale entre les échantillons, la compilation des résultats a été basée non pas sur les valeurs mesurées des paramètres $L^{*}$ et $b^{*}$ de l'échelle Hunter Lab mais sur le taux de leurs variations par rapport à l'état initial. Ce taux, noté $T_{i}$ (où $\mathrm{i}=L^{*}$ ou $\left.b^{*}\right)$ a été défini comme étant : $T_{i}=$ $\left[\left(i_{O}-i\right) / i_{O}\right]$, où $T_{i}$ est le taux de variation du paramètre $i$ à l'instant $t, i_{O}$ étant égal à la valeur initiale du paramètre et $i$ étant la valeur du paramètre $i$ mesurée à l'instant $t$.

\subsection{Effet relatif de la température et de l'oxygène sur le changement de couleur}

Une analyse de variance a été effectuée afin de déterminer l'effet relatif de la température et de la concentration en oxygène sur la variation du paramètre $b^{*}$ de l'échelle Hunter Lab au cours de l'évolution de la couleur des dattes durant le stockage.

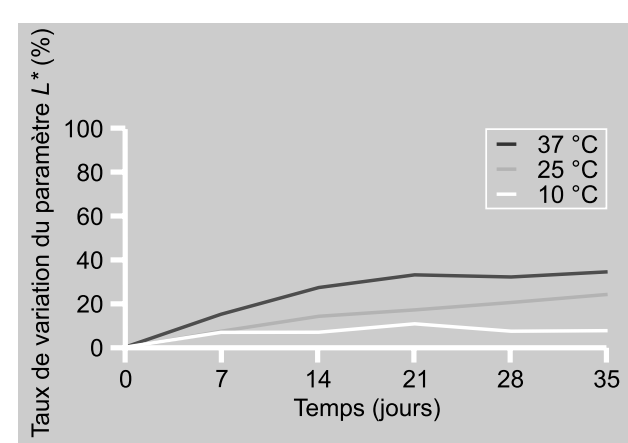

Figure 1.

Mesure du paramètre $L^{*}$ de l'échelle Hunter Lab mesurant l'évolution de la couleur de dattes Deglet Nour durant l'entreposage d'échantillons placés en sachets contenant de l'atmosphère à $40 \%$ d'oxygène, en fonction de trois températures de stockage différentes.

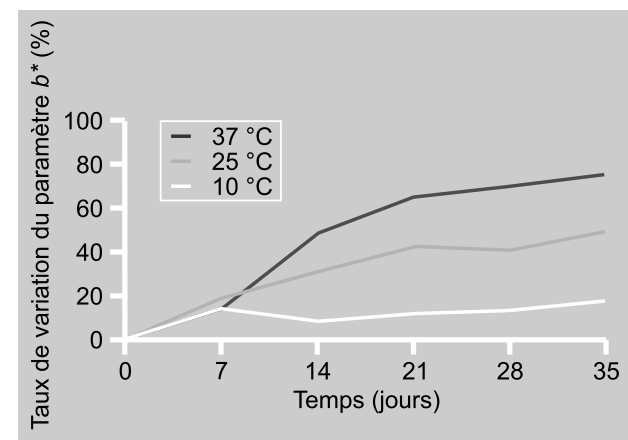

Figure 2.

Mesure du paramètre $b^{*}$ de l'échelle Hunter Lab mesurant l'évolution de la couleur de dattes Deglet Nour durant l'entreposage d'échantillons placés en sachets contenant de l'atmosphère à $40 \%$ d'oxygène, en fonction de trois températures de stockage différentes.

\section{Résultats et discussion}

\section{1. Évolution de la couleur durant l'entreposage}

Pour une concentration en oxygène de $40 \%$, le taux de variation du paramètre $L^{*}$ n'a jamais dépassé $30 \%$ (figure 1) alors que, pour le paramètre $b^{*}$, le taux de variation a pu atteindre des valeurs supérieures à $70 \%$ (figure 2). Cela tendrait à prouver que, même si la luminosité de la couleur des dattes est affectée pendant l'entreposage, c'est le virage de la couleur jaune dorée vers la couleur sombre qui constitue le phénomène le plus significatif. Ce virage a été visuellement détecté à partir d'un taux de variation de $46 \%$ pour le paramètre $L^{*}$ et de $68 \%$ pour le paramètre $b^{*}$.

Des constatations analogues ont été observées en présence des deux autres concentrations d'oxygène (20\% et $0 \%$ ) testées (figures 3 à 0 ). Par ailleurs, l'analyse des courbes de variations des paramètres $L^{*}$ et 
Figure 3.

Mesure du paramètre $L^{*}$ de l'échelle Hunter Lab mesurant l'évolution de la couleur de dattes Deglet Nour durant l'entreposage d'échantillons placés en sachets contenant de l'atmosphère à $20 \%$ d'oxygène, en fonction de trois températures de stockage différentes.

Figure 4.

Mesure du paramètre $b^{*}$ de l'échelle Hunter Lab mesurant l'évolution de la couleur de dattes Deglet Nour durant l'entreposage d'échantillons placés en sachets contenant de l'atmosphère à $20 \%$ d'oxygène, en fonction de trois températures de stockage différentes.

Figure 5

Mesure du paramètre $L^{*}$ de l'échelle Hunter Lab mesurant l'évolution de la couleur de dattes Deglet Nour durant l'entreposage d'échantillons placés en sachets sous vide (0 \% d'oxygène), en fonction de trois températures de stockage différentes.

Figure 6.

Mesure du paramètre $b^{*}$ de l'échelle Hunter Lab mesurant l'évolution de la couleur de dattes Deglet Nour durant l'entreposage d'échantillons placés en sachets sous vide ( $0 \%$ d'oxygène), en fonction de trois températures de stockage différentes.

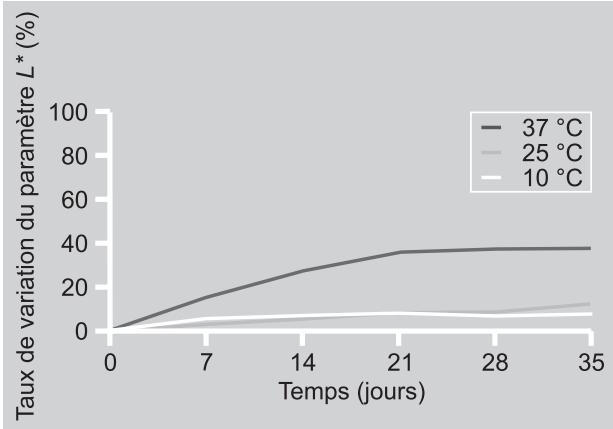

$b^{*}$ au cours du temps (figures 1 à $O$ ) a montré que plus la température augmentait, plus la variation des paramètres était significative. L'élévation de la température aurait donc bien un effet sur la cinétique de dégradation de la couleur de la datte. Cet effet s'est révélé d'autant plus prononcé que la concentration d'oxygène augmentait, ce qui appuierait l'hypothèse du déclenchement d'un brunissement non enzymatique oxydatif que nous avons précédemment évoqué. Les deux courbes de variation des paramètres $L^{*}$ ou $b^{*}$ correspondant à des températures de stockage de $25^{\circ} \mathrm{C}$ et $10^{\circ} \mathrm{C}$ sont apparues presque confondues dans le cas des sachets d'échantillons mis sous vide ou placés en atmosphère à $20 \%$ d'O ${ }_{2}$ (figures 3 à G) ; cependant, ces deux mêmes courbes ont été très distinctes de celles mesurées sur les échantillons en sachets contenant $40 \%$ d'O$_{2}$ (figures 1 et 2 ). L'interaction entre les facteurs température et taux d'oxygène se révèle statistiquement non significative (tableau I). Par ailleurs, d'après les figures 5 et 6 , l'absence totale d'oxygène n'a pas empêché la dégradation de la couleur durant l'entreposage ; la dégradation de la couleur serait donc partiellement causée par un brunissement enzymatique ayant lieu à basse ou moyenne température.

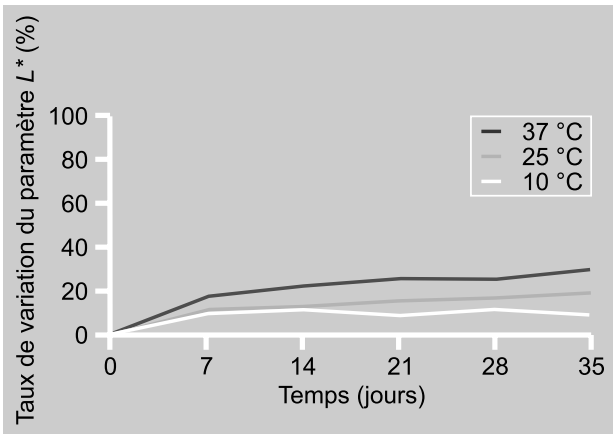

\subsection{Effet relatif de la température et de l'oxygène sur le changement de couleur}

Statistiquement, seule la température a eu un effet significatif sur la variation du paramètre $b^{*}$ de l'échelle Hunter Lab intervenant dans la caractérisation de la couleur des dattes au cours du stockage (tableau I) ; cet effet a été très significatif puisque, pour un seuil de signification de $5 \%$, la valeur de $F_{\text {exp }}(73,74)$ a été largement supérieure à celle de $F_{\text {th }}(4,26)$. Le taux de contribution, qui reflète la part de variation de la couleur expliquée par le changement de température, est d'environ $89 \%$

\section{Conclusion}

Nos résultats ont montré que l'évolution des dattes au cours de leur stockage peut être 
Tableau I.

Effet relatif de la température $\left[(10,25 \text { ou } 37)^{\circ} \mathrm{C}\right]$ et de la concentration en oxygène $[(0,20$ ou 40$) \%]$ sur la variation du paramètre $b^{*}$ de l'échelle Hunter Lab au cours de l'évolution de la couleur de dattes Neglet Nour entreposées (Tunisie).

\begin{tabular}{lccrrrrrr}
\hline Source de variation & $\begin{array}{c}\text { Degrés } \\
\text { de liberté }\end{array}$ & $\begin{array}{c}\text { Somme des } \\
\text { carrés des écarts }\end{array}$ & Variance & $F_{\text {exp }}$ & $F_{\text {th }}$ & Signification & $\%$ de contribution \\
Température & 2 & 9568,75 & 4784,37 & 73,74 & 4,26 & Significatif & 88,8 \\
Oxygène & 2 & 92,86 & 46,43 & 0,71 & 4,26 & Non significatif & 0,9 \\
Interaction & 4 & 530,32 & 132,58 & 2,04 & 3,63 & Non significatif & 4,9 \\
Variation résiduelle & 9 & 583,88 & 64,88 & - & - & - & 5,4
\end{tabular}

suivie par l'étude de la couleur dominante du fruit. Cette couleur se révèle pouvoir être prédite à partir du seul paramètre $b^{*}$ de l'échelle Hunter Lab. L'analyse de variance à deux facteurs effectuée a montré que, dans le cas des dattes conditionnées sous différentes concentrations en oxygène et à différentes températures, la perte de la couleur initiale du fruit était davantage expliquée par une augmentation de la température ambiante que par la variation de la concentration en oxygène du milieu de conservation. Notre étude a aussi mis en évidence que, durant l'entreposage à différentes températures, deux métabolismes pouvaient avoir lieux : un brunissement enzymatique et un brunissement non enzymatique oxydatif des dattes. Cependant, le brunissement non enzymatique du type de la réaction de Maillard n'a pas été important car les températures d'entreposage testées n'ont pas été favorables à ce type de réaction.

\section{Références}

[1] Reynes M., Étude filière dattes. Mission FAO. Volet traitement post-récolte et qualité des dattes, Cirad-Flhor, Doc. Interne, Montpellier, 1995.
[2] Barreveld W.H., Date palm products, FAO Agricultural Services, Bull. No. 101, Roma, Italy, 1993.

[3] Rygg G.L., The relation of moisture content to rate of darkening in Deglet Nour dates, Date Growers' Institute 34 (1957) 476-485.

[4] Achour M., Ben Amara S., Ben Salem N., Jebali A., Hamdi M., Effet des conditionnements sous vide et sous atmosphère modifiée sur la conservation des dattes Deglet Nour tunisiennes, Fruits 58 (4) (2003) 205212.

[5] Bourgois C.M., La conservation sous atmosphère modifiée, in: Microbiologie alimentaire, tome 1, Aspect microbiologique de la sécurité et la qualité alimentaires, Tec\&Doc Lavoisier, $2^{e}$ éd., Paris, France, 1990, pp. 395-406.

[6] Navarro S., Storage of dates under carbon dioxide atmosphere for quality preservation, in: Proc. Int. Conf. Controlled Atmosphere and Fumigation in Stored Products, Exclusive Printing Services, Clovis, CA, USA, 2001, pp. 231-239.

[7] Calero F.A., Active packaging and color control: the case of fruit and vegetables, in: Novel Food Packaging Techniques, 1st ed., Woodhead Publ. Ltd, Cambrige, England, 2003, pp. 416-438.

[8] Hong J.H., Gross K.G., Maintaining quality of fresh-cut tomato slices through modified atmosphere packaging and low temperature storage, J. Food Sci. 66 (7) (2001) 75-80. 


\section{Efecto de las condiciones de almacenamiento en la degradación del color de los dátiles tunecinos de tipo Deglet Nour.}

Resumen - Introducción. En Túnez, los dátiles de tipo Deglet Nour son famosos por su color amarillo dorado y tienen un alto valor de mercado; constituyen la mayoría de los dátiles exportados hacia Europa. El objetivo de nuestro trabajo consistió en estudiar el efecto del oxígeno y la temperatura en la degradación del color de este tipo de dátiles durante el período de almacenamiento. Material y métodos. La evolución del color de dátiles de tipo Deglet Nour almacenados bajo humedad relativa constante $(75 \%)$, a tres temperaturas distintas $\left[(10,25 \text { y } 37)^{\circ} \mathrm{C}\right]$ y en presencia de tres concentraciones de oxígeno $[(0,20$ y 40)\%], se siguió cada semana, durante 35 días, mediante la medición de los parámetros $L^{*}$ y $b^{*}$ de la escala Hunter Lab. Resultados. La temperatura desempeñó un papel determinante en la degradación del color de los dátiles durante el almacenamiento; el efecto del oxígeno fue importante aunque secundario. Conclusión. Se observa que el color de los dátiles durante su almacenamiento puede predecirse contando sólo con el parámetro $b^{*}$ de la escala Hunter Lab. En el caso de los dátiles acondicionados con diferentes concentraciones de oxígeno y a distintas temperaturas, la pérdida del color inicial del fruto se explica más por un aumento de la temperatura ambiente que por la variación de la concentración de oxígeno del medio de conservación. La degradación del color vendría dada por pardeamientos no enzimáticos y enzimáticos oxidativos.

Túnez / Phoenix dactylífera / dátil / color / almacenamiento / reacciones bioquímicas / biodegradación

To access this journal online: www.edpsciences.org 